\title{
Radiation-induced meningioma following radiation therapy for pituitary adenoma
}

\section{Case report}

\section{Mark K. Lyons, M.D., Gilbert R. Gonzales, M.D., Steven E. Schild, M.D., and Kent D. Nelson,} M.D.

Departments of Neurological Surgery, Neurology, Radiation Oncology, and Diagnostic Radiology, Mayo Clinic, Scottsdale, Arizona

The authors report the long-term result of treatment of a presumed pituitary adenoma with external-beam radiation therapy, which appears to be the development of a radiation-induced meningioma. Of the post radiation neoplasms that have been reported, meningiomas constitute a large proportion of these lesions.

Key Words * radiation-induced meningioma * pituitary adenoma

\section{CASE REPORT}

This 27-year-old woman presented in June 1970 with a 2-year history of decreasing vision. Ophthalmological examination revealed bilateral optic pallor and bitemporal hemianopsia. A large pituitary adenoma $(5.5 \mathrm{~cm})$ was diagnosed by cerebral angiography and pneumoencephalography. Surgical resection was recommended. The patient refused surgery and was treated instead with 4075 cGy of external-beam radiation in 24 fractions consisting of $6 \mathrm{MV}$ x-rays and cobalt- 60 gamma rays.

Treatment was delivered with 8 X 8-cm opposed lateral fields. The patient was started and maintained on a course of steroid and thyroid supplement. She was followed until 1979 with serial neurological and ophthalmological examinations and, once it became available, with computerized tomography (CT). No change in the size of the pituitary tumor was noted throughout this time period and the patient continued to refuse additional medical or surgical therapy. She was lost to follow-up evaluation until 1995.

In 1995, 25 years after her radiation treatment, she sought medical evaluation at our institution for a 2-year history of mental status deterioration, personality change, and visual loss. Neurological examination was notable for a decline in higher intellectual function, afferent pupillary defects, apraxia, dysconjugate gaze, and frontal signs. Ophthalmological examination confirmed bilateral optic neuropathy with dense right homonymous field defect and no significant left eye vision. Her endocrinological findings were consistent with a nonfunctioning adenoma. She had continued her course of steroid and thyroid replacement therapy since 1970. She had not had any imaging studies since 1980. A magnetic resonance imaging study was obtained that demonstrated the $5-\mathrm{cm}$ mass arising from the sellar region and a 6-cm lesion arising immediately adjacent to the pituitary mass (Fig. 1). Although there 
did not appear to be any change in the size of the original pituitary mass (in comparison to previous CT scans), the adjacent mass was new. The imaging characteristics of this new mass were most consistent with meningioma. Surgical resection of the tumors was recommended, but the patient and family refused treatment.

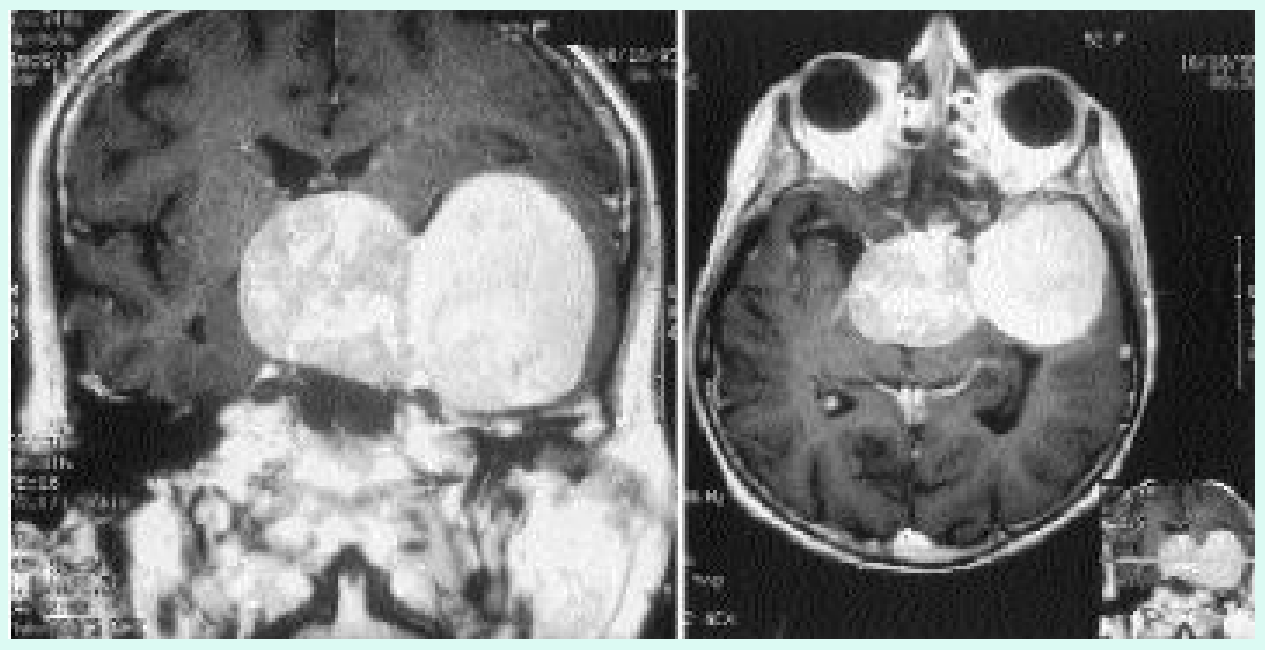

Fig. 1. Coronal (left) and axial (right) T1-weighted magnetic resonance images with gadolinium enhancement, demonstrating an inhomogeneous 5-cm mass that appears to arise from the sella turcica and extend superiorly into the suprasellar cistern elevating and almost obliterating the anterior aspect of the third ventricle, deforming the midbrain and upper pons. There may be some invasion of the left cavernous sinus. Immediately adjacent to the pituitary mass in the anterior middle cranial fossa is a uniformly enhancing 6-cm mass demonstrating a typical T2 signal decrease on long TR/long TE sequence consistent with a meningioma. The tumor displaces the middle cerebral artery and temporal lobe superiorly. The supraclinoid internal carotid artery courses between the two tumors.

Radiation-induced tumors are well reported in the literature. To be considered a radiation-induced lesion, the tumor must fulfill the following criteria: 1 ) it must occur in the field of radiation; 2) there must be a delay of several years from the time of treatment to its appearance (usually 3-25 years); and 3) the induced lesion must differ histologically from the original tumor that was irradiated. In our case, the first two requirements were met. However, no histological diagnosis was ever made for either lesion. Magnetic resonance imaging is becoming more sophisticated allowing for more precise preoperative assessment of diagnostic possibilities. The two lesions display distinctly different imaging characteristics and arguably could fulfill the third requirement for radiation-induced tumors, despite the absence of a histological confirmation. It is possible that future advances in imaging technology will afford even more preoperative insight into tumor pathology. This case demonstrates the major features of radiation-induced tumors. Continued vigilance in these patients is important, particularly in the face of changing or developing symptoms.

Manuscript received May 21, 1996.

Accepted in final form June 18, 1996.

Address reprint requests to: Mark K. Lyons, M.D., Department of Neurological Surgery, Mayo Clinic Scottsdale, 13400 East Shea Boulevard, Scottsdale, Arizona 85259. 